Historic, Archive Document

Do not assume content reflects current scientific knowledge, policies, or practices. 

PRICE LIST

Effective June 1, 1920

\section{DYBVIG NURSERY}

\section{The Home of the Little Evergreens}

COLTON, S. D.

Prices quoted are prepaid

Everything guaranteed

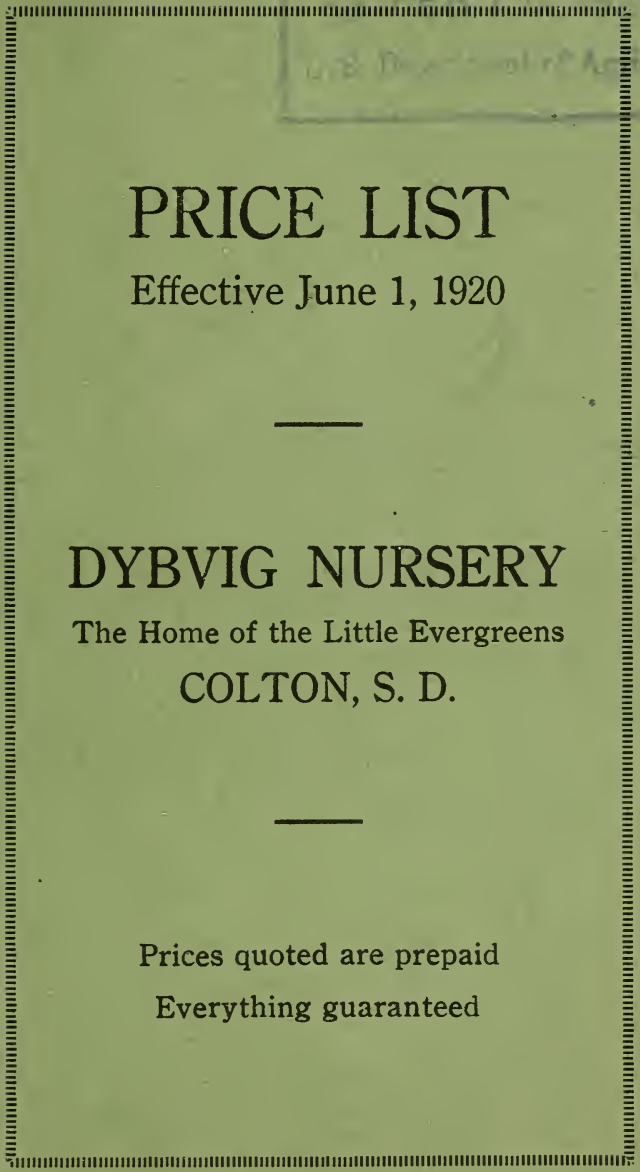



PRICE LIST EFFECTIVE JUNE 1, 1920

\section{DYBVIG NURSERY}

The Home of the Little Evergreens

COLTON, :: :: S. D.

\section{EVERGREENS}

All Pines- Each Dozen Hundred

$12-18 " \ldots \ldots .60$

$18-24 " \ldots .00$

$\$ 6.00$

$\$ 40.00$

$2-3^{\prime} \ldots \ldots \ldots \ldots$

9.00

60.00

12.00

90.00

Black Hills Spruce-

$12-18^{\prime \prime} \ldots \ldots \ldots 1.00$

10.00

70.00

$18-24$ " _.

12.00

90.00

$2-3^{\prime} \ldots \ldots \ldots \ldots 2.00$

20.00

Colcrado Green Spruce-
$12-18 "$ "-_-_- 1.25
$18-24 " \ldots \ldots 1.50$
12.00
15.00
(Balled and Burlapped)

90.00

Colorado Blue Spruce-Seìect-

\begin{tabular}{|c|c|c|c|}
\hline $18-24 " \ldots$ & 5.00 & 50.00 & $-\ldots$ \\
\hline $24-30 " \ldots$ & 7.50 & 75.00 & $\ldots$ \\
\hline $30-36 " \ldots$ & 10.00 & 100.00 & \\
\hline $3-4^{\prime}----$ & 12.50 & 120.00 & \\
\hline$-5^{\prime}---$ & 15.00 & 150.00 & \\
\hline$-6^{\prime}--$. & 20.00 & ---- & \\
\hline$-8^{\prime} \ldots--$ & 30.00 & ---- & \\
\hline$-10^{\prime}$ & 50.00 & $\ldots$ & \\
\hline
\end{tabular}


Colorado Green Spruce-

$\mathrm{EACH}$

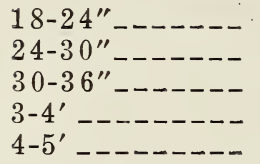

3.50

4.00

5.00

7.00

10.00
DOZEN

36.00

$45.0 \mathrm{O}$

55.00

75.00

110.00
PER 100

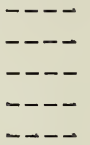

35.00

50.00

72.00

110.00

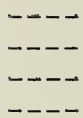

3.50

4.50

6.50

10.00

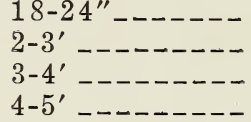

Rlack Hills Pine-
3.50

4.00

6.50

10.00
36.00

45.00

72.00

110.00

\section{Douglas Spruce-}

\begin{tabular}{|c|c|c|}
\hline $3-4^{\prime}-------$ & 7.00 & 75.00 \\
\hline $\begin{array}{l}4-5^{\prime}-------- \\
5-6^{\prime}\end{array}$ & 10.00 & 110.00 \\
\hline $\begin{array}{l}5-6^{\prime}-\cdots \\
6-8^{\prime}-\cdots\end{array}$ & $\begin{array}{l}15.00 \\
20.00\end{array}$ & -- \\
\hline
\end{tabular}

\section{Scotch \& Austrian Pine-}

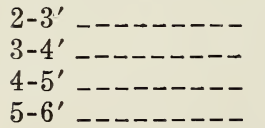

4.00

45.00

6.50

72.00

10.00

12.00

\section{Dwarf Mountain Pine-}
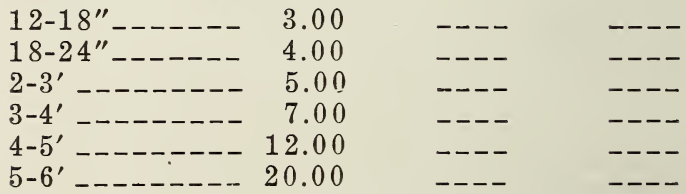


\section{WRUI'T TREES}

Apples and Crabs-
$\mathrm{EACH}$
DOZEN
PER 100

5-6'_-_-_-_ 1.50

18.00

4-5'_-_-_- 1.25

15.00

$3-4^{\prime}-\ldots+----1.00$

12.00

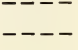

$---$

Plums-

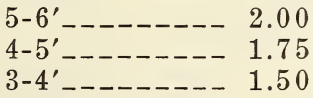

20.00

18.00

15.00

$---$

$---$

Cherries-

Sand 2-3'_-- 1.00

10.00

Other Varieties-

4-5' - - $5-2.00$

20.00

25.00

$----$

\section{Grapes-}

Beta _-_-_-_-_ _-

Common _..- ..-

10.00

6.00

- - -

$---$

Currants-

Common _-_-_ _-_ Special-Perfection Gooseberries-

Common _-_- .75

Raspberries-

Common _... - .-

St. Regis_-_- _--

Sunbeam -..- ..-

Ohta --.-.-. -.-

Minn. No. 4 --

Black Caps_- -.-

Blackberries - ---

2.50

12.00

3.00

20.00

3.00

20.00

4.00

35.00

5.00

50.00

3.00

12.00

3.00

12.00

Dewberries- - -- 
Strawberries-

$\mathrm{EACH}$

DOZEN

PER 100

Common

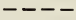

3.00

Everbearing -_ _-_

Asparagus-_- - --

Rhubarb- - - - -

$\overline{1.5} 0$

6.00

4.50

7.50

Horseradish- ---

3.00

25.00

20.00

\section{ORNAMENTAL TREES}

Ash, Box Elder, Soft Maple-

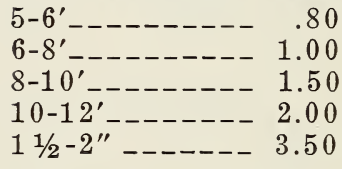

8.00

100.00

10.00

60.00

15.00

80.00

20.00

100.00

25.00

125.00

Elms-Common-

5-6'_-_-

8.00

60.00

$6-8^{\prime} \ldots \ldots \ldots \ldots 1.25$

12.00

90.00

$8-10^{\prime} \ldots \ldots \ldots-1.75$

18.00

125.00

$10-12^{\prime}+\ldots \ldots-02.25$

22.00

$11 / 2-2$ " _._. 3.00

30.00

175.00

Elms - Special street tree stock headed as per catalog-

$11 / 2-2 "$
$2-21 / 2 "-\ldots-$
$21 / 2-3 "$

40.00

5.00

50.00

60.00

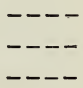

Linden-

$5-6^{\prime}$

15.00

$6-8^{\prime}-\ldots-----2.00$

20.00

$8-10^{\prime}+\ldots \ldots+-\ldots 2.50$

25.00

Butternut \& Walnut-

$5-6^{\prime}+\ldots-\ldots-\ldots-1.50$

12.00

$6-8^{\prime}-\ldots-----2.00$

20.00

8-10'__-_-_- 2.50

25.00 
Poplars-Carolina, Canadian, Norway-

$\mathrm{EACH}$

6-8'-1.--

$8-10^{\prime}$

Boolena-
DOZEN

8.00

10.00
PER 100

60.00

80.00

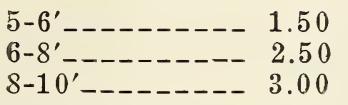

Silver Leaf-

$5-6^{\prime}-1.75$
$6-8^{\prime}-\ldots-\cdots-1.00$

7.20

10.00

$---$

- - -

Balm of Gilead-

$5-6{ }^{\prime}-\ldots------1.00$

10.00

6-8'

15.00

Hackberry-Native-

$5-6{ }^{\prime} \ldots \ldots \ldots-\ldots 1.00$

10.00

$6-8^{\prime}-\ldots-\ldots-1.50$

15.00

$8-10^{\prime}+\ldots \ldots-\ldots-2.00$

20.00

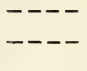

$---$

\section{Hard Maple-}

$5-6{ }^{\prime}+\ldots-----1.50$

15.00

$6-8^{\prime}+\ldots-\ldots-0-2.00$

18.00

Mountain Ash-

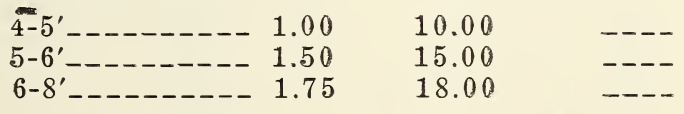

\section{Cut Leaf Birch-}
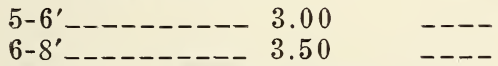

$----$

Weeping Mountain Ash-

5-6' 
Niobe Willow-

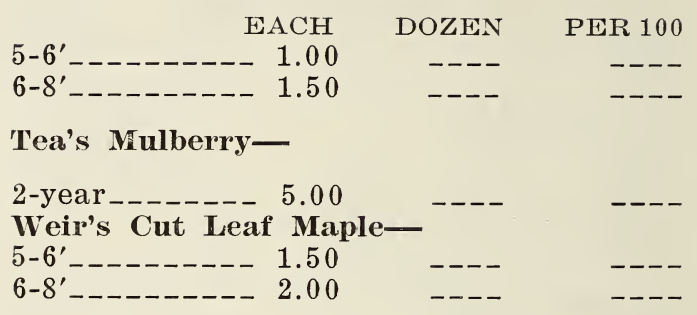

\section{SEEDLINGS}

Ash, Box Elder, Elm, Soft Maple-

Per 100 Per 1000

$12-18 " \ldots \ldots 2.50$ \$ 20.00

$18-24 "$ "-_-_-_-_-_ $4.00 \quad 30.00$

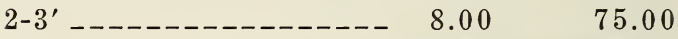

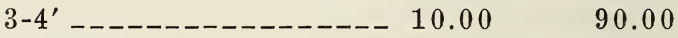

Poplars-

Norway and Carolina-

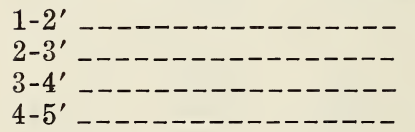

Canadian-

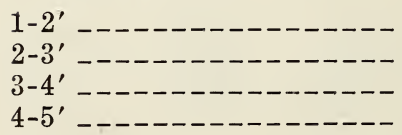

5.00

50.00

8.00

70.00

12.00

100.00

All Willows-

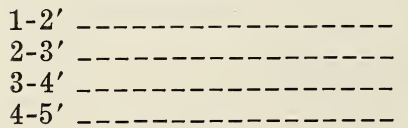

4.00

35.00

6.00

50.00

9.50

90.00

12.00

100.00 


\section{CUTTINGS}

Willows, Poplars, Cottonwoods-

_-

\section{HEDGE STOCK}

Buckthorn, Caragana, Russian Olive-

$12-18$ " - .

$18-24 "$ -

$2-3^{\prime}+\ldots \ldots \ldots \ldots$

Barberry-

$12-18 " \ldots \ldots+0.00$

Dogivood, Spirea, HoneysuckleEACH DOZEN

PER 100

2-3'

$\$ 6.00$

$3 \times 4^{\prime}-\ldots-\ldots-\ldots-\ldots$

9.00

$\$ 40.00$ 60.00

\section{FIOWERING SHRUBS}

Cornus Siberica

Yellow Flowering Currant

Elderberry

Sumach

Syringa Grandiflora

Syringa Brandiflora

High Bush Cranberry

Honeysuckle Tartarian

Lilac-Purple

Barberry Thumbergii

Spirea Arguta

Spirea Van Houti

Snowball

Snowberry

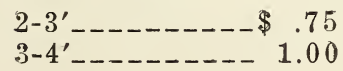

8.00

10.00 
Other Varieties-

$\begin{array}{rrr}\text { EACH } & \text { DOZEN } & \text { PER } 100 \\ 2-33^{\prime}-------1.00 & 10.00 & --- \\ 3-4^{\prime}--------1.25 & 12.00 & ---\end{array}$

\section{Spirea-Van Houti-}

Large clumps that will bloom the

$$
\text { first year_-- } 2.50 \quad 25.00
$$

Perian Lilacs-

Clumps _-_--- 2.50

Named Lilacs-

$2-3^{\prime}+\ldots \ldots+\ldots-1.25$

$3-4$ '

Roses-

Climbing _..- $\mathbf{1 . 5 0}$

Rugosa _...- 1.25

H. P. - - - - 2.00

Hardy Per-

ennial Plants .50

Bleeding Heart 1.00

Yucca _-_._- . .75

Phlox ----- .50

Lilies _-

Lilies of

the Valley_- .25

Iris _..._. . .50

Peonies in

variety _.-- 1.00

Dahlias _..._. .40

Gladiolus _... - .-

Tulips - - - - - - --

Climbing Vines .75

Clematis

Large flowering 1.50 Others _._-_ 1.00
12.00

15.00

$---$

$--\cdots$

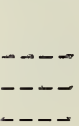

5.00

10.00

7.50

5.00

5.00

2.50

5.00

$---1$

$-\infty-\infty$

10.00

4.00

1.25

8.00

1.50

7.20

10.00

15.00

10.00 

